OPEN ACCESS

Edited by:

Bin Jiang,

Peking University, China

Reviewed by: Junjie Ma,

Amgen, United States Xiaomeng Yue,

University of Cincinnati, United States

${ }^{*}$ Correspondence:

Jing Yuan

jyuan@fudan.edu.cn

†These authors have contributed equally to this work

Specialty section:

This article was submitted to Family Medicine and Primary Care,

a section of the journal

Frontiers in Medicine

Received: 25 April 2021

Accepted: 20 May 2021

Published: 17 June 2021

Citation:

Li M, Lv G, Hsieh S, Shao R and Yuan J (2021) Pandemic Worry and Preventive Health Behaviors During

the COVID-19 Outbreak.

Front. Med. 8:700072.

doi: 10.3389/fmed.2021.700072

\section{Pandemic Worry and Preventive Health Behaviors During the COVID-19 Outbreak}

\author{
Minghui $\mathrm{Li}^{1+}$, Gang $\mathrm{Lv}^{2 \dagger}$, Stephanie Hsieh ${ }^{3}$, Rongjie Shao ${ }^{4}$ and Jing Yuan ${ }^{5 *}$ \\ ${ }^{1}$ Department of Clinical Pharmacy and Translational Science, University of Tennessee Health Science Center, Memphis, TN, \\ United States, 2 Department of General Surgery, The First Medical Center of Chinese PLA General Hospital, Beijing, China, \\ ${ }^{3}$ Department of Pharmacy, Scarborough Health Network-Centenary Hospital, Scarborough, ON, Canada, ${ }^{4}$ Department of \\ Health Economics, China Pharmaceutical University, Nanjing, China, ${ }^{5}$ Department of Clinical Pharmacy and Pharmacy \\ Administration, Fudan University School of Pharmacy, Shanghai, China
}

Objective: As schools are preparing for onsite learning, it is urgently needed to characterize the extent of pandemic worry and to examine predictors of adopting preventive health behaviors of hand washing, face mask wearing, and maintaining social distance among student pharmacists.

Methods: An online survey was sent to 326 student pharmacists in the United States. Pandemic worry was measured using a seven-point Likert scale ranging from extremely not afraid of, to extremely afraid of getting COVID-19. The health belief model (HBM) was the theoretical framework of this study. Preventive health behaviors and components of the HBM were also measured using seven-point Likert scales (one indicated extremely unlikely; seven indicated extremely likely). Multivariable linear regression models were used to identify predictors of each behavior.

Results: A medium level of pandemic worry $(M=4.2, S D=1.92)$ was identified and females reported a higher pandemic worry. Respondents reported that they were extremely likely to wash their hands $(M=6.8, S D=0.48)$ and maintain social distance $(M=6.6, S D=0.92)$, but were moderately unlikely to wear face masks $(M=2.2, S D=$ 1.51). Determinants of face mask wearing included pandemic worry, perceived benefits, cue to action, self-efficacy, and being of an Asian American. Perceived barriers were negatively associated with face mask wearing.

Conclusion: Strategies should be implemented to reduce the psychological impact of COVID-19 pandemic among student pharmacists. Predictors identified in this study should be incorporated in efforts to improve face mask wearing. Continued monitoring of pandemic worry and preventive health behaviors is of great significance when universities and colleges are for onsite learning.

Keywords: COVID-19, pandemic worry, hand washing, face mask wearing, maintaining social distance 


\section{INTRODUCTION}

Coronavirus disease 2019 (COVID-19) is a new respiratory illness caused by SARS-CoV-2 coronavirus. It was first detected in Wuhan, China in December 2019 and has rapidly spread worldwide (1). COVID-19 was declared as a pandemic by the World Health Organization (WHO) in March 2020 (2). The first US case of COVID-19 was reported in Washington State on January 19, 2020 (3). From the initial clusters identified in the states of Washington, California, and New York, confirmed cases of COVID-19 quickly expanded to all 50 states (4). The federal government implemented Coronavirus Guidelines and put forth certain preventative measures to slow the spread of COVID-19, such as maintaining good hand hygiene, avoiding social gatherings, closing schools, and non-essential businesses in areas with evidence of community transmission. Subsequently, multiple state and local officials implemented stay-at-home orders. Despite these containment efforts, the number of confirmed cases of COVID-19 continued to rise nationally (5). The United States has now the highest number of COVID-19 infections and has become the new epicenter of the pandemic (1).

The COVID-19 outbreak has generated significant pandemic worry. Pandemic worry refers to the constant, uncontrollable, and unwanted negative thoughts about the future outcomes of disease (6). These thoughts might impede individuals' problem-solving skills, prevent engagement in protective health behaviors, and contribute to the development of anxiety and depression. College students might be particularly vulnerable to the psychological impacts as they face pressure in many aspects of life, such as in academics and employment, especially given their immaturely developed psychological system. With the current COVID-19 outbreak, significant pandemic worry has been reported among college students in China $(7,8)$. This psychological impact might be due to the consequences of national and local measures taken during the outbreak, including school closures and rising unemployment rates secondary to lockdowns. Colleges and universities in the United States have implemented certain preventive measures, such as closures of campuses, cancellation of experiential rotations, changes in the delivery of didactic courses and exams, which might adversely affect the psychological well-being of college students (9). Negative psychological consequences due to the abruptness and timing of these implementations near the end of the academic school year include graduation delays, jeopardization of campus housing, and meal programs security, poor adaptation to the new learning style and exam delivery, and inability to continue with lectures for those with resource constraints.

Individuals' preventive health behaviors are important to contain the spread of COVID-19. The health belief model (HBM) is a theoretical framework that has been widely used in previous infectious disease outbreaks to evaluate individuals' motivations to adopt preventive health behaviors (10-14). Key components of the HBM include perceived susceptibility (how likely the individual perceives they will get the disease), perceived severity (individuals' perception of the severity of the illness), perceived benefits (individuals' belief about the effectiveness of the health behavior), perceived barriers (individuals' belief of the obstacles that prevent them from adopting the health behavior), cue to action (an external stimulus that triggers an individual to adopt the behavior), and self-efficacy (individuals' confidence of their ability to engage in the behavior) (15). In the 2002 to 2003 Severe Acute Respiratory Syndrome (SARS) epidemic, perceived susceptibility, perceived benefits, and cue to action significantly predicted face mask wearing and hand washing in Hong Kong $(10,11)$. In the $2009 \mathrm{H} 1 \mathrm{~N} 1$ pandemic, perceived susceptibility, perceived severity, perceived benefits, and cue to action determined the adoption of maintaining social distance and hand washing in South Korea (12). For face mask wearing during the H1N1 pandemic, perceived susceptibility, perceived benefits, perceived barriers, and cue to action were significant predictors in Hong Kong (14).

To slow the spread of COVID-19, the Center for Disease Control and Prevention (CDC) has recommended that individuals adopt certain preventive health behaviors. These recommendations include hand washing with soap and water or hand sanitizer, keeping a six-feet social distance from other people, and covering the mouth and nose with a cloth face mask when around others (16). The guidance on face mask wearing was modified in April 2020 in light of recent evidence suggesting that transmission of the SARS-CoV-2 coronavirus might still occur among asymptomatic and pre-symptomatic individuals (17). Because these individuals comprise of a significant proportion of people infected with COVID-19, the CDC revised previous recommendation that face masks should only be worn by sick or caregivers of sick individuals, to the new recommendation that everyone should wear a cloth face mask in public settings when social distancing is hard to maintain.

To the best of our knowledge, no studies have evaluated the impact of pandemic worry on the adoption of preventive health behaviors during the pandemic of COVID-19 among student pharmacists. Health professional students are facing a high risk of COVID-19 infections through their interning, practicing, and working environments. To fill the gap in the literature, the objectives of this study were: (1) to characterize the extent of pandemic worry and (2) to examine predictors of adopting CDCendorsed preventive health behaviors, including hand washing, face mask wearing, and maintaining social distance, among student pharmacists.

\section{METHODS}

An online survey was sent to 326 student pharmacists enrolled in a PharmD program in the south of the United States. Both those attended didactic course and engaged in experiential learning were surveyed in this study. A pilot study of five respondents was conducted before the formal study to make sure the clarity of survey questions. The survey was distributed in March 2020 and finished in 2 weeks. During that time, the number of COVID19 cases continued to grow in the United States. Following the federal Coronavirus Guidelines and state and local stay-at-home orders, most colleges, and universities were closed and didactic courses were adjusted to virtual learning. In addition, preceptors at some clinical sites were unable to take student pharmacists who 
are considered non-essential. Thus, most clinical rotations were rescheduled or canceled.

Information collected in this study included demographics, academic setting, pandemic worry, knowledge, and preventive health behaviors. Demographic information collected included age, gender, and race. Didactic course and experiential learning were two academic settings studied. Pandemic worry was measured by frequency (seven-point Likert scale, from never to always worried about getting COVID-19) and severity (sevenpoint Likert scale, from extremely not afraid of to extremely afraid of getting COVID-19). Knowledge of COVID-19 was measured by the source of information, availability of test kit, medication, and vaccine, and perceived knowledge (seven-point Likert scale, from extremely low to extremely high).

CDC-endorsed preventive health behaviors, including hand washing, face mask wearing, and maintaining social distance, were examined in this study (16). The likelihood of performing these preventive health behaviors was reported using sevenpoint Likert scales, where one indicated extremely unlikely and seven indicated extremely likely. Key components of the HBM, including perceived susceptibility, perceived severity, perceived benefits, perceived barrier, cue to action, and self-efficacy, were also measured using seven-point Likert scales.

The distributions of demographics, academic setting, pandemic worry, and knowledge were reported by frequency and percentage. Means of pandemic worry, knowledge, and preventive health behaviors were analyzed and compared by different age, gender, race, and academic setting groups using $t$-tests or analysis of variance (ANOVA) tests. The correlations between different preventive health behaviors were measured using Pearson correlation coefficients. Multivariable linear regression models were used to identify predictors of hand washing, face mask wearing, and maintaining social distance. In each regression model, the preventive health behavior was the dependent variable and pandemic worry, knowledge, components of the HBM, demographics, and academic setting were independent variables. SAS version 9.4 (SAS Institute, Cary, NC) was used to perform all statistical analyses.

\section{RESULTS}

Among 326 respondents who completed the survey, the majority of them were aged 18-26 years (79\%), female (74\%), and Caucasian (68\%). The distribution between different academic settings at the time of survey completion was approximately equal, including $53 \%$ attending didactic course and $47 \%$ engaged in experiential learning (Table 1). Most respondents reported hearing about COVID-19 through digital channels such as social media (73\%), TV (43\%), and websites (40\%). Respondents reported a medium level of pandemic worry severity $(M=$ $4.2, S D=1.92)$. The availability of a test kit $(92 \%)$, lack of availability of approved medications (74\%), and lack of availability of a vaccine (93\%) for COVID-19 were correctly identified by the majority of respondents (Table 1). Respondents reported a slightly high perceived knowledge of COVID-19 $(M=4.8, S D=1.03)$. Females reported a significantly lower knowledge compared to males ( 4.7 vs. $5.2, p<0.001$ ), and those attended didactic course reported a significantly lower knowledge compared to those engaged in experiential learning (4.7 vs. 4.9, $p=0.03$; Table 2).

Respondents reported a medium level of pandemic worry severity $(M=4.2, S D=1.92)$. Females reported a significantly higher worry severity compared to males (4.4 vs. $3.7, p=$ 0.004). No differences between worry severity were found among students of different ages, races, and academic settings (Table 2). For the pandemic worry frequency in the past week, $51 \%$ of respondents reported that they worried about getting COVID-19 less than half the time and 37\% of respondents reported more than half the time. Specifically, $7 \%$ of respondents reported always worried about getting COVID-19 in the past week (Table 1).

Hand washing was positively correlated with face mask wearing $(r=0.14, p=0.01)$ and maintaining social distance $(r=0.26, p<0.001)$. However, face mask wearing was not correlated with maintaining social distance $(r=0.10, p=0.07)$. Respondents reported that they were extremely likely to wash their hands $(M=6.8, S D=0.48)$ and maintain social distance $(M=6.6, S D=0.92)$, but were moderately unlikely to wear a face mask in public $(M=2.2, S D=1.51)$. Females were significantly more likely to wash hands compared to males (6.9 vs. 6.7, $p=$ 0.006). Asian Americans were significantly more likely to wear a face mask compared to other racial groups (Caucasian: 2.1, African American: 2.0, Asian American: 3.0, Other: 2.1, $p=$ 0.004; Table 2).

Respondents reported a slightly high perceived susceptibility to COVID-19 $(M=4.7, S D=1.79)$. The perceived susceptibility was different among different racial groups (Caucasian: 4.8, African American: 4.6, Asian American: 4.0, Other: 5.5, $p$ $=0.006$ ) and those attended didactic course perceived a significantly lower susceptibility compared to those engaged in experiential learning (4.4 vs. 5.1, $p<0.001$ ). Respondents reported a moderately high perceived severity of COVID-19 ( $M$ $=5.8, S D=1.13)$. Perceived benefits were moderately high for hand washing $(M=6.1, S D=1.01)$ and maintaining social distance $(M=6.2, S D=0.97)$, but were medium for face mask wearing $(M=3.5, S D=1.71)$. Perceived barriers were slightly high for face mask wearing $(M=4.9, S D=1.88)$, slightly low for hand washing $(M=3.4, S D=2.04)$, and moderately low for maintaining social distance $(M=2.4, S D=1.47)$. Cue to action was extremely high for hand washing $(M=6.7, S D=$ $0.57)$, moderately high for maintaining social distance $(M=$ $6.4, S D=0.97)$, and slightly high for face mask wearing $(M=$ $5.3, S D=1.75)$. Females reported a significantly higher cue to action of hand washing ( 6.8 vs. $6.5, p=0.003)$ and a significantly higher cue to action of maintaining social distance ( 6.5 vs. 6.2, $p=0.03)$ compared to males. Self-efficacy was extremely high for hand washing $(M=6.5, S D=0.87)$, moderately high for maintaining social distance $(M=6.2, S D=1.15)$, and slightly low for face mask wearing $(M=3.4, S D=1.98)$. Those attended didactic course reported a significantly higher self-efficacy of hand washing (6.6 vs. $6.4, p=0.01$ ) and a significantly higher self-efficacy of maintaining social distance (6.3 vs. $6.0, p=0.02$ ) compared to those engaged in experiential learning (Table 2 ). 
TABLE 1 | Demographics, academic setting, pandemic worry, and knowledge of study participants $(N=326)$.

\begin{tabular}{|c|c|c|}
\hline & $N$ & $\%$ \\
\hline \multicolumn{3}{|l|}{ Demographics } \\
\hline \multicolumn{3}{|l|}{ Age } \\
\hline $18-26$ & 258 & 79.1 \\
\hline $27+$ & 68 & 20.9 \\
\hline \multicolumn{3}{|l|}{ Gender } \\
\hline Female & 242 & 74.2 \\
\hline Male & 84 & 25.8 \\
\hline \multicolumn{3}{|l|}{ Race } \\
\hline Caucasian & 222 & 68.1 \\
\hline African American & 40 & 12.3 \\
\hline Asian American & 45 & 13.8 \\
\hline Other & 19 & 5.8 \\
\hline \multicolumn{3}{|l|}{ Academic setting } \\
\hline Didactic course & 172 & 52.8 \\
\hline Experiential learning & 154 & 47.2 \\
\hline \multicolumn{3}{|l|}{ Pandemic worry } \\
\hline \multicolumn{3}{|l|}{ Frequency } \\
\hline Never & 30 & 9.2 \\
\hline Occasionally & 74 & 22.7 \\
\hline Sometimes & 63 & 19.3 \\
\hline About half the time & 37 & 11.3 \\
\hline Often & 59 & 18.1 \\
\hline Most of the time & 41 & 12.6 \\
\hline Always & 22 & 6.7 \\
\hline \multicolumn{3}{|l|}{ Knowledge } \\
\hline \multicolumn{3}{|l|}{ Source } \\
\hline TV & 140 & 42.9 \\
\hline Website & 129 & 39.6 \\
\hline Social media & 239 & 73.3 \\
\hline Radio & 22 & 6.7 \\
\hline In print & 23 & 7.1 \\
\hline Family/friend & 136 & 41.7 \\
\hline \multicolumn{3}{|l|}{ Availability of test kit } \\
\hline Yes & 299 & 91.7 \\
\hline No & 17 & 5.2 \\
\hline Unsure & 10 & 3.1 \\
\hline \multicolumn{3}{|c|}{ Availability of medication } \\
\hline Yes & 33 & 10.1 \\
\hline No & 242 & 74.2 \\
\hline Unsure & 51 & 15.6 \\
\hline \multicolumn{3}{|l|}{ Availability of vaccine } \\
\hline Yes & 5 & 1.5 \\
\hline No & 303 & 92.9 \\
\hline Unsure & 18 & 5.5 \\
\hline
\end{tabular}

Perceived severity $(\beta: 0.08 ; 95 \%$ CI: $0.04-0.13)$ and cue to action $(\beta$ : $0.21 ; 95 \%$ CI: $0.11-0.30)$ were predictors for hand washing. Being a male was negatively associated with hand washing ( $\beta$ : $-0.13 ; 95 \%$ CI: -0.24 to -0.01$)$. Determinants of face mask wearing included pandemic worry $(\beta$ : $0.18 ; 95 \%$
CI: $0.10-0.26)$, perceived benefits ( $\beta$ : $0.29 ; 95 \%$ CI: $0.21-0.38)$, cue to action ( $\beta$ : $0.10 ; 95 \% \mathrm{CI}: 0.01-0.18)$, self-efficacy ( $\beta$ : 0.18 ; 95\% CI: 0.11-0.25), and being of an Asian American ( $\beta$ : 0.57; 95\% CI: 0.17-0.97). Perceived barriers were negatively associated with face mask wearing ( $\beta$ : $-0.12 ; 95 \% \mathrm{CI}:-0.20$ to -0.05$)$. Cue to action ( $\beta$ : $0.38 ; 95 \%$ CI: $0.28-0.49)$ and self-efficacy $(\beta$ : 0.13 ; $95 \% \mathrm{CI}$ : $0.04-0.23)$ were predictors for maintaining social distance (Table 3).

\section{DISCUSSION}

This study found that student pharmacists had a medium level of pandemic worry severity and females had a higher worry severity than males. Pandemic worry might be associated with adverse psychological outcomes and students are more vulnerable. Cao et al. (7) found that $25 \%$ of college students reported experiencing anxiety amidst the COVID-19 outbreak in China. Wang et al. (8) reported that moderate or severe psychological impact was experienced by $54 \%$ of the general population in China. Females and students were significant predictors of experiencing greater psychological impact amidst the COVID-19 outbreak (8). Student pharmacists might be more psychologically vulnerable due to their younger age, the challenges they face with the transition into adulthood and to the profession (e.g., managing finances, adapting to new learning regimes, making new friends, and creating a new identity as a pharmacist). The abrupt change in their daily lives associated with strict government measures to contain the outbreak (e.g., school and non-essential business closures), the consequences of these measures (e.g., financial pressure to pay tuition expenses amidst uncertain employment prospect and social isolation from friends), and the rapid changes in the social and economic climate during the COVID-19 outbreak, might further compound their pandemic worry.

Additional psychosocial and economic factors that disproportionately affect females, such as low social status, income inequality, gender-based violence, and caregiving responsibility, might explain the higher level of pandemic worry observed (18). Potential protective factors of pandemic worry during the COVID-19 outbreak include social support and living with parents (7). Therefore, strategies such as creating virtual peer support groups, organizing virtual activities, and encouraging students to maintain contact with their family and friends virtually, might help to mitigate the psychological impact of COVID-19. If appropriate and feasible, student pharmacists might also consider moving back with their parents during the COVID-19 outbreak.

Student pharmacists reported their knowledge of COVID-19 to be slightly high. Improvement of knowledge, dissemination of additional health information, provision of regular updates on the latest information, and accessibility of information via reliable sources, have been identified as protective factors against adverse psychological outcomes during the COVID-19 outbreak (8). Websites and social media proved to be effective strategies for getting knowledge to student pharmacists based on our results. Therefore, increasing education and providing regular updates through government websites or social media accounts 
TABLE 2 | Pandemic worry, knowledge, and preventive health behaviors of study participants by age, gender, race, and academic setting.

\begin{tabular}{|c|c|c|c|c|c|c|c|c|c|c|c|c|c|c|c|}
\hline & \multirow{3}{*}{$\begin{array}{l}\frac{\text { Total }}{} \\
M(S D)^{\dagger}\end{array}$} & \multicolumn{3}{|c|}{ Age } & \multicolumn{3}{|c|}{ Gender } & \multicolumn{5}{|c|}{ Race } & \multicolumn{3}{|c|}{ Academic setting } \\
\hline & & $18-26$ & $27+$ & \multirow[t]{2}{*}{$p$ value } & Female & Male & \multirow[t]{2}{*}{$p$ value } & Caucasian & $\begin{array}{l}\text { African } \\
\text { American }\end{array}$ & \multirow{2}{*}{$\begin{array}{c}\text { Asian } \\
\text { American }\end{array}$} & Other & \multirow[t]{2}{*}{$p$ value } & \multirow{2}{*}{$\begin{array}{l}\begin{array}{l}\text { Didactic } \\
\text { Course }\end{array} \\
M(S D)\end{array}$} & \multirow{2}{*}{$\begin{array}{c}\text { Experiential } \\
\text { Learning }\end{array}$} & \multirow[t]{2}{*}{$p$ value } \\
\hline & & $M(S D)$ & $M(S D)$ & & $M(S D)$ & $M(S D)$ & & $M(S D)$ & $M(S D)$ & & $M(S D)$ & & & & \\
\hline Pandemic worry & $4.2(1.92)$ & $4.2(1.93)$ & $4.3(1.90)$ & 0.50 & $4.4(1.87)$ & $3.7(1.96)$ & $0.004^{*}$ & $4.1(1.92)$ & $4.1(2.00)$ & $4.8(1.83)$ & $4.5(1.81)$ & 0.13 & $4.2(1.88)$ & $4.2(1.97)$ & 0.85 \\
\hline Knowledge & $4.8(1.03)$ & $4.8(1.00)$ & $4.7(1.16)$ & 0.33 & $4.7(1.02)$ & $5.2(0.99)$ & $<0.001^{*}$ & $4.9(0.96)$ & $4.7(1.09)$ & $4.5(1.18)$ & $4.6(1.34)$ & 0.14 & $4.7(1.04)$ & $4.9(1.02)$ & $0.03^{*}$ \\
\hline \multicolumn{16}{|l|}{ Preventive health behavior } \\
\hline Hand washing & $6.8(0.48)$ & $6.8(0.43)$ & $6.8(0.62)$ & 0.53 & $6.9(0.44)$ & $6.7(0.56)$ & $0.006^{\star}$ & $6.8(0.50)$ & $6.9(0.35)$ & $6.8(0.53)$ & $6.9(0.32)$ & 0.34 & $6.8(0.48)$ & $6.8(0.48)$ & 0.51 \\
\hline Face mask wearing & $2.2(1.51)$ & $2.1(1.45)$ & $2.3(1.74)$ & 0.37 & $2.2(1.49)$ & $2.2(1.59)$ & 0.89 & $2.1(1.46)$ & $2.0(1.27)$ & $2.9(1.91)$ & $2.1(0.97)$ & $0.004^{\star}$ & $2.2(1.51)$ & $2.1(1.52)$ & 0.72 \\
\hline Maintaining social distance & $6.6(0.92)$ & $6.6(0.94)$ & $6.6(0.85)$ & 0.86 & $6.6(0.91)$ & $6.5(0.96)$ & 0.20 & $6.6(0.98)$ & $6.5(1.06)$ & $6.8(0.47)$ & $6.5(0.70)$ & 0.46 & $6.6(0.79)$ & $6.5(1.05)$ & 0.16 \\
\hline Perceived susceptibility & $4.7(1.79)$ & $4.7(1.81)$ & $4.8(1.75)$ & 0.64 & $4.7(1.80)$ & $4.8(1.77)$ & 0.59 & $4.8(1.73)$ & $4.6(1.84)$ & $4.0(1.94)$ & $5.5(1.54)$ & $0.006^{*}$ & $4.4(1.87)$ & $5.1(1.62)$ & $<0.001^{\star}$ \\
\hline Perceived severity & $5.8(1.13)$ & $5.8(1.08)$ & $5.9(1.29)$ & 0.80 & $5.9(1.07)$ & $5.7(1.27)$ & 0.17 & $5.8(1.12)$ & $5.9(1.03)$ & $6.0(0.95)$ & $5.4(1.64)$ & 0.27 & $5.8(1.19)$ & $5.9(1.05)$ & 0.17 \\
\hline \multicolumn{16}{|l|}{ Perceived benefits } \\
\hline Hand washing & $6.0(1.01)$ & $6.1(1.01)$ & $5.9(1.00)$ & 0.27 & $6.1(1.00)$ & $5.9(1.02)$ & 0.11 & $6.0(0.99)$ & $6.2(1.08)$ & $6.0(1.00)$ & $6.1(1.18)$ & 0.85 & $6.0(1.04)$ & $6.1(0.98)$ & 0.83 \\
\hline Face mask wearing & $3.5(1.71)$ & $3.5(1.68)$ & $3.8(1.81)$ & 0.24 & $3.6(1.70)$ & $3.3(1.73)$ & 0.09 & $3.5(1.72)$ & $3.6(1.75)$ & $3.9(1.76)$ & $3.3(1.33)$ & 0.43 & $3.4(1.67)$ & $3.6(1.75)$ & 0.29 \\
\hline Maintaining social distance & $6.2(0.97)$ & $6.3(0.94)$ & $6.1(1.06)$ & 0.13 & $6.3(0.96)$ & $6.1(0.98)$ & 0.12 & $6.2(0.99)$ & $6.2(1.11)$ & $6.5(0.73)$ & $6.5(0.77)$ & 0.19 & $6.3(0.95)$ & $6.2(0.98)$ & 0.20 \\
\hline \multicolumn{16}{|l|}{ Perceived barrier } \\
\hline Hand washing & $3.4(2.04)$ & $3.4(2.05)$ & $3.3(2.04)$ & 0.56 & $3.4(2.03)$ & $3.5(2.09)$ & 0.72 & $3.5(2.01)$ & $3.0(2.17)$ & $3.7(2.10)$ & $2.7(1.89)$ & 0.18 & $3.3(1.97)$ & $3.6(2.12)$ & 0.23 \\
\hline Face mask wearing & $4.9(1.88)$ & $4.9(1.87)$ & $4.8(1.93)$ & 0.91 & $4.8(1.90)$ & $5.1(1.82)$ & 0.26 & $4.9(1.83)$ & $4.5(2.09)$ & $5.0(2.09)$ & $4.6(1.50)$ & 0.54 & $4.8(1.80)$ & $5.0(1.96)$ & 0.31 \\
\hline Maintaining social distance & $2.4(1.47)$ & $2.4(1.47)$ & $2.1(1.49)$ & 0.17 & $2.3(1.51)$ & $2.4(1.37)$ & 0.84 & $2.3(1.43)$ & $2.4(1.56)$ & $2.4(1.63)$ & $2.4(1.54)$ & $>0.99$ & $2.4(1.49)$ & $2.3(1.46)$ & 0.58 \\
\hline \multicolumn{16}{|l|}{ Cue to action } \\
\hline Hand washing & $6.7(0.57)$ & $6.7(0.58)$ & $6.7(0.55)$ & 0.93 & $6.8(0.48)$ & $6.5(0.75)$ & $0.003^{\star}$ & $6.7(0.52)$ & $6.8(0.42)$ & $6.6(0.83)$ & $6.5(0.70)$ & 0.20 & $6.7(0.60)$ & $6.7(0.55)$ & 0.88 \\
\hline Face mask wearing & $5.3(1.75)$ & $5.3(1.73)$ & $5.3(1.83)$ & 0.91 & $5.4(1.64)$ & $5.1(2.00)$ & 0.13 & $5.3(1.76)$ & $5.5(1.60)$ & $5.7(1.53)$ & $4.6(2.19)$ & 0.12 & $5.3(1.79)$ & $5.3(1.71)$ & 0.85 \\
\hline Maintaining social distance & $6.4(0.97)$ & $6.4(0.97)$ & $6.4(0.95)$ & 0.93 & $6.5(0.87)$ & $6.2(1.18)$ & $0.03^{*}$ & $6.4(0.87)$ & $6.5(0.91)$ & $6.6(0.89)$ & $5.9(1.85)$ & 0.07 & $6.5(1.03)$ & $6.4(0.90)$ & 0.49 \\
\hline \multicolumn{16}{|l|}{ Self-efficacy } \\
\hline Hand washing & $6.5(0.87)$ & $6.5(0.74)$ & $6.3(1.25)$ & 0.19 & $6.5(0.89)$ & $6.4(0.83)$ & 0.54 & $6.5(0.79)$ & $6.5(1.09)$ & $6.4(1.16)$ & $6.7(0.45)$ & 0.61 & $6.6(0.68)$ & $6.4(1.03)$ & $0.01^{*}$ \\
\hline Face mask wearing & $3.4(1.98)$ & $3.4(1.96)$ & $3.5(2.04)$ & 0.86 & $3.3(1.95)$ & $3.8(2.04)$ & 0.06 & $3.5(1.93)$ & $3.2(1.99)$ & $3.7(2.17)$ & $2.8(2.01)$ & 0.39 & $3.6(1.97)$ & $3.2(1.97)$ & 0.13 \\
\hline Maintaining social distance & $6.2(1.15)$ & $6.2(1.07)$ & $6.0(1.39)$ & 0.17 & $6.2(1.16)$ & $6.1(1.12)$ & 0.37 & $6.1(1.18)$ & $6.3(1.01)$ & $6.4(0.87)$ & $5.9(1.56)$ & 0.26 & $6.3(0.95)$ & $6.0(1.32)$ & $0.02^{\star}$ \\
\hline
\end{tabular}

${ }_{1} 1$ as extremely low; 7 as extremely high.

${ }^{*} p<0.05$. 
TABLE 3 | Factors associated with preventive health behaviors.

\begin{tabular}{|c|c|c|c|c|c|c|c|c|c|}
\hline Pandemic worry & \multicolumn{3}{|c|}{ Hand washing } & \multicolumn{3}{|c|}{ Face mask wearing } & \multicolumn{3}{|c|}{ Maintaining social distance } \\
\hline Knowledge & 0.02 & -0.03 & 0.06 & -0.03 & -0.16 & 0.11 & -0.02 & -0.10 & 0.07 \\
\hline \multicolumn{10}{|l|}{ Health belief model } \\
\hline Perceived susceptibility & 0.03 & 0.00 & 0.06 & -0.01 & -0.09 & 0.07 & 0.03 & -0.03 & 0.08 \\
\hline Perceived benefits & 0.03 & -0.02 & 0.08 & 0.29 & 0.21 & 0.38 & -0.03 & -0.13 & 0.06 \\
\hline Perceived barriers & 0.00 & -0.03 & 0.02 & -0.12 & -0.20 & 0.05 & -0.06 & -0.13 & 0.00 \\
\hline Cue to action & 0.21 & 0.11 & 0.30 & 0.10 & 0.01 & 0.18 & 0.38 & 0.28 & 0.49 \\
\hline Self-efficacy & 0.06 & 0.00 & 0.12 & 0.18 & 0.11 & 0.25 & 0.13 & 0.04 & 0.23 \\
\hline \multicolumn{10}{|l|}{ Demographics } \\
\hline Female & Ref & & & Ref & & & Ref & & \\
\hline Male & -0.13 & -0.24 & -0.01 & 0.21 & -0.11 & 0.53 & 0.02 & -0.18 & 0.23 \\
\hline \multicolumn{10}{|l|}{ Ethnicity } \\
\hline Caucasian & Ref & & & Ref & & & Ref & & \\
\hline African American & 0.10 & -0.05 & 0.25 & -0.09 & -0.50 & 0.32 & -0.11 & -0.37 & 0.16 \\
\hline Asian American & 0.01 & -0.14 & 0.15 & 0.57 & 0.17 & 0.97 & 0.13 & -0.13 & 0.39 \\
\hline Other & 0.18 & -0.03 & 0.39 & 0.03 & -0.56 & 0.61 & 0.21 & -0.16 & 0.59 \\
\hline \multicolumn{10}{|l|}{ Academic setting } \\
\hline Didactic course & Ref & & & Ref & & & Ref & & \\
\hline Experiential learning & 0.02 & -0.08 & 0.12 & 0.00 & -0.28 & 0.28 & -0.10 & -0.28 & 0.08 \\
\hline
\end{tabular}

could be used to mitigate the psychological impact associated with COVID-19.

Student pharmacists reported that they were extremely likely to wash their hands and maintain social distance, but were moderately unlikely to wear a face mask. Given the low willingness to wear a face mask, predictors identified in our study should be considered to encourage face mask wearing. Components of the HBM that predicted face mask wearing were perceived benefits, perceived barriers, cue to action, and selfefficacy. Perceived benefits and cue to action as predictors of face mask wearing were consistent with previous studies conducted in Asia (10-12). Barriers to face mask wearing identified in previous studies included discomfort (e.g., difficulty with breathing), inconvenience (e.g., need to remove masks during meal times), forgetfulness, and social acceptance (19). Strategies should be taken to improve face mask wearing following the recent CDC guidelines revision. For example, government, public health officials, and healthcare professionals can actively promote face mask wearing, as well as improve the public's knowledge on the effectiveness of face masks in reducing COVID-19 transmission. By widely promoting face mask wearing, this can increase social acceptance and reduce stigmatization and discrimination against people who wear them (20). Messaging should also make clear of the recommendations for the use of cloth masks and to not use medical masks reserved for medical professionals (17).
Videos on how to make cloth masks can be created by public health officials or organizations and be uploaded on official government websites and YouTube, in order to improve selfefficacy of face mask use. YouTube, due to its considerable reach, has recently been identified as an under-tapped platform for promoting preventive health behaviors amidst the COVID-19 pandemic (21). As this study also identified cue to action as a predictor of hand washing and maintaining social distance, public messaging should continue to emphasize the importance of hand washing and maintaining social distancing, along with face mask wearing, in containing the spread of COVID-19.

There are several strengths of this study. First, this is the first study evaluating pandemic worry among student pharmacists. The results of this study highlighted the vulnerability of student pharmacists to the psychological impact of the pandemic and emphasize the need to implement strategies to mitigate this effect. Second, predictors of face mask wearing could be used to improve face mask use among the public in light of recent expansion in CDC guidelines. Third, this study applied the theoretical framework of the health belief model to systematically assess the determinants of preventive health behaviors. This could inform public health initiatives to contain the COVID-19 outbreak when universities and colleges reopen for onsite learning. This study has some limitations worth noting. First, the survey was conducted prior to $\mathrm{CDC}$ modifying recommendations on face 
mask wearing. Respondents' perception of face mask wearing reported might change in light of the new recommendations. Second, the survey collected information at a single point in time. Thus, it cannot capture changes in the students' pandemic worry and preventive health behaviors as the COVID-19 pandemic evolves. Third, this study relied on students' self-reports using a survey. It might not reflect their actual level of pandemic worry and preventive health behaviors due to social desirability. Fourth, this study only included student pharmacists in the study population. The results might not be generalizable to other health professional students.

\section{CONCLUSION}

The COVID-19 pandemic has generated a medium-level of pandemic worry among student pharmacists in the United States. Females reported a higher level of pandemic worry than males. Strategies should be implemented to reduce the psychological impact of COVID-19 pandemic. For preventive health behaviors, student pharmacists reported that they were moderately unlikely to use a face mask and were extremely likely to engage in hand washing and social distancing. The health belief model components that predicted face mask wearing (i.e., perceived benefits, perceived barriers, cue to action, and selfefficacy) should be incorporated in efforts to improve face mask wearing following CDC's recent expansion of face mask recommendations. Continued monitoring of pandemic worry and preventive health behaviors among student pharmacists are

\section{REFERENCES}

1. World Health Organization. Coronavirus Disease (COVID-2019) Situation Reports. Available online at: https://www.who.int/emergencies/diseases/ novel-coronavirus-2019/situation-reports

2. World Health Organization. Rolling Updates on Coronavirus Disease (COVID19). Available online at: https://www.who.int/emergencies/diseases/novelcoronavirus-2019/events-as-they-happen

3. Holshue ML, DeBolt C, Lindquist S, Lofy KH, Wiesman J, Bruce H, et al. First case of 2019 Novel Coronavirus in the United States. N Engl J Med. (2020) 382:929-36. doi: 10.1056/NEJMoa2001191

4. Omer SB, Malani P, Del Rio C. The COVID-19 pandemic in the US: a clinical update. JAMA. (2020) 323:1767-8. doi: 10.1001/jama.2020.5788

5. Centers for Disease Control Prevention. Cases in the U.S. Available online at https://www.cdc.gov/coronavirus/2019-ncov/cases-updates/casesin-us.html

6. Scherr CL, Jensen JD, Christy K. Dispositional pandemic worry and the health belief model: promoting vaccination during pandemic events. J Public Health. (2017) 39:e242-e50. doi: 10.1093/pubmed/fdw101

7. Cao W, Fang Z, Hou G, Han M, Xu X, Dong J, et al. The psychological impact of the COVID-19 epidemic on college students in China. Psychiatry Res. (2020) 287:112934. doi: 10.1016/j.psychres.2020.112934

8. Wang C, Pan R, Wan X, Tan Y, Xu L, Ho CS, et al. Immediate psychological responses and associated factors during the initial stage of the 2019 coronavirus disease (COVID-19) Epidemic among the general population in China. Int J Environ Res Public Health. (2020) 17:1729. doi: 10.3390/ijerph17051729

9. Bayham J, Fenichel EP. Impact of school closures for COVID-19 on the US health-care workforce and net mortality: a modelling study. Lancet Public Health. (2020) 5:e271-e8. doi: 10.1016/S2468-2667(20)30082-7 of great significance when universities and colleges are preparing for onsite learning.

\section{DATA AVAILABILITY STATEMENT}

The raw data supporting the conclusions of this article will be made available by the authors, without undue reservation.

\section{ETHICS STATEMENT}

The studies involving human participants were reviewed and approved by University of Tennessee Health Science Center. The patients/participants provided their written informed consent to participate in this study.

\section{AUTHOR CONTRIBUTIONS}

ML and GL: conceptualization, methodology, and writing. SH: methodology and writing. RS: methodology and analysis. JY: conceptualization, methodology, analysis, writing, and supervision. All authors contributed to the article and approved the submitted version.

\section{ACKNOWLEDGMENTS}

The authors thank all participants of this study and Arlesha Armstrong for her assistance with data collection.

10. Tang CS, Wong CY. Factors influencing the wearing of facemasks to prevent the severe acute respiratory syndrome among adult Chinese in Hong Kong. Prev Med. (2004) 39:1187-93. doi: 10.1016/j.ypmed.2004. 04.032

11. Wong CY, Tang CS. Practice of habitual and volitional health behaviors to prevent severe acute respiratory syndrome among Chinese adolescents in Hong Kong. J Adolesc Health. (2005) 36:193-200. doi: 10.1016/j.jadohealth.2004.02.024

12. Jeong HS, Lee DW, Youn CH, Lee MK, Lee SJ, Suh YS, et al. Perception and performance of preventive behaviors for the pandemic influenza in hospital employees and outpatients. Yonsei Med J. (2011) 52:181-7. doi: $10.3349 /$ ymj.2011.52.1.181

13. Coe AB, Gatewood SB, Moczygemba LR, Goode JV, Beckner JO. The use of the health belief model to assess predictors of intent to receive the novel (2009) H1N1 influenza vaccine. Innov Pharm. (2012) 3:1-11. doi: 10.24926/iip.v3i2.257

14. Zhang CQ, Chung PK, Liu JD, Chan DKC, Hagger MS, Hamilton K. Health beliefs of wearing facemasks for influenza A/H1N1 prevention: a qualitative investigation of Hong Kong older adults. Asia Pac J Public Health. (2019) 31:246-56. doi: 10.1177/1010539519844082

15. Rosenstock IM, Strecher VJ, Becker MH. Social learning theory and the Health Belief Model. Health Educ Q. (1988) 15:175-83. doi: $10.1177 / 109019818801500203$

16. Centers for Disease Control and Prevention. How to Protect Yourself \& Others. Available online at: https:/www.cdc.gov/coronavirus/2019-ncov/ prevent-getting-sick/prevention.html

17. Centers for Disease Control and Prevention. Recommendation Regarding the Use of Cloth Face Coverings, Especially in Areas of Significant CommunityBased Transmission. Available online at: https://www.cdc.gov/coronavirus/ 2019-ncov/prevent-getting-sick/cloth-face-cover.html 
18. Faravelli C, Alessandra Scarpato M, Castellini G, Lo Sauro C. Gender differences in depression and anxiety: the role of age. Psychiatry Res. (2013) 210:1301-3. doi: 10.1016/j.psychres.2013. 09.027

19. Sim SW, Moey KS, Tan NC. The use of facemasks to prevent respiratory infection: a literature review in the context of the Health Belief Model. Singapore Med J. (2014) 55:160-7. doi: 10.11622/smedj. 2014037

20. Feng S, Shen C, Xia N, Song W, Fan M, Cowling BJ. Rational use of face masks in the COVID-19 pandemic. Lancet Respir Med. (2020) 8:434-6. doi: 10.1016/S2213-2600(20) 30134-X

21. Basch CH, Hillyer GC, Meleo-Erwin ZC, Jaime C, Mohlman J, Basch CE. Preventive behaviors conveyed on YouTube to mitigate transmission of COVID-19: cross-sectional study. JMIR Public Health Surveill. (2020) 6:e18807. doi: 10.2196/18807

Conflict of Interest: The authors declare that the research was conducted in the absence of any commercial or financial relationships that could be construed as a potential conflict of interest.

Copyright (c) $2021 \mathrm{Li}, \mathrm{Lv}$, Hsieh, Shao and Yuan. This is an open-access article distributed under the terms of the Creative Commons Attribution License (CC BY).

The use, distribution or reproduction in other forums is permitted, provided the original author(s) and the copyright owner(s) are credited and that the original publication in this journal is cited, in accordance with accepted academic practice. No use, distribution or reproduction is permitted which does not comply with these terms. 